\title{
70
}

\section{ISOS: Intelligent Shell Of SNMP}

\author{
Jianxin LI, Benjamin J. LEON \\ University of Southwestern Louisiana, U.S.A.
}

SNMP is today's dominant network management software product. In this poster, we propose an approach to enhance the functions of SNMP through the use of an intelligent shell. The shell concept in network management is akin to that in operating systems. ISOS uses the shell script to aggregate SNMP operations. It supports the imperative features, such as sequencing, alternation, and iteration. In addition, ISOS incorporates searching and planning techniques to support query manipulation and agent-oriented programming. We claim that ISOS can relieve a network manager from tedious monitoring and controlling of the network, and it can also reduce the management traffic overload. Our prototype of ISOS is dependent on Unix shell and SNMPv2 developed by Carnegie-Mellon University. 